\title{
Experimental validation of a model of an uncontrolled bicycle
}

\author{
J.D.G. Kooijman • A.L. Schwab · J.P. Meijaard
}

Received: 22 September 2006 / Accepted: 26 February 2007 /

Published online: 5 May 2007

(C) Springer Science+Business Media, Inc. 2007

\begin{abstract}
In this paper, an experimental validation of some modelling aspects of an uncontrolled bicycle is presented. In numerical models, many physical aspects of the real bicycle are considered negligible, such as the flexibility of the frame and wheels, play in the bearings, and precise tire characteristics. The admissibility of these assumptions has been checked by comparing experimental results with numerical simulation results.

The numerical simulations were performed on a three-degree-of-freedom benchmarked bicycle model. For the validation we considered the linearized equations of motion for small perturbations of the upright steady forward motion. The most dubious assumption that was validated in this model was the replacement of the tires by knife-edge wheels rolling without slipping (non-holonomic constraints).

The experimental system consisted of an instrumented bicycle without rider. Sensors were present for measuring the roll rate, yaw rate, steering angle, and rear wheel rotation. Measurements were recorded for the case in which the bicycle coasted freely on a level surface. From these measured data, eigenvalues were extracted by means of curve fitting. These eigenvalues were then compared with the results from the linearized equations of motion of the model. As a result, the model appeared to be fairly accurate for the low-speed low-frequency behaviour.
\end{abstract}

Keywords Bicycle dynamics · Experiments · Instrumentation · Multibody dynamics · Non-holonomic constraints

J.D.G. Kooijman · A.L. Schwab ( $ه)$

Laboratory for Engineering Mechanics, Delft University of Technology, Mekelweg 2, 2628 CD Delft, The Netherlands

e-mail: a.l.schwab@tudelft.nl

J.P. Meijaard

School of MMME, The University of Nottingham, University Park, Nottingham NG7 2RD, UK

e-mail: jaap.meijaard@nottingham.ac.uk 
Fig. 1 The bicycle model: four rigid bodies (rear wheel, rear frame, front handlebar assembly, front wheel) connected by three revolute joints (rear hub, steering axis, front hub), together with the coordinate system, the degrees of freedom, and the parameters

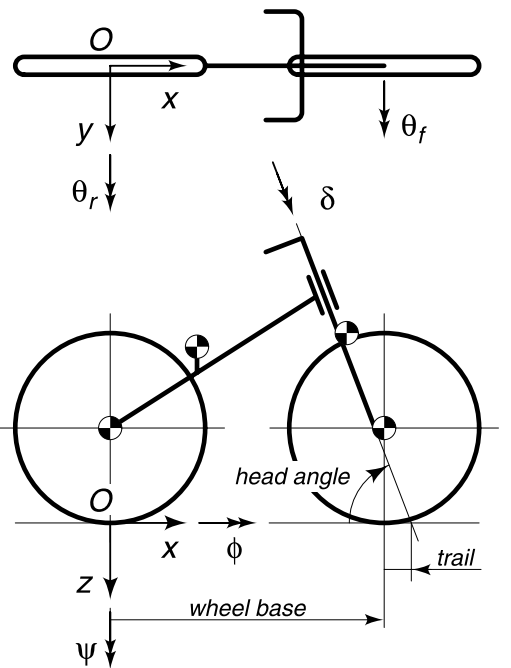

\section{Introduction}

The governing dynamic equations for a general bicycle model have recently been benchmarked [17] and after more than a century of bicycle dynamics literature we are confident about the correctness. In this model, many physical aspects of the real bicycle are considered negligible, such as the flexibility of the frame and wheels, play in the bearings, and precise tire characteristics. The admissibility of these assumptions is checked by comparing experimental results with numerical simulation results. Apart from flexibility and play, the most dubious assumption to be validated in this model is the replacement of the tires by knife-edge wheels rolling without slipping (non-holonomic constraints).

The bicycle model, shown in Fig. 1, consists of four rigid bodies connected by revolute joints. The contact between the knife-edge wheels and the flat level surface is modelled by holonomic constraints in the normal direction and by non-holonomic constraints in the longitudinal and lateral directions. In the absence of a rider (or with a rider rigidly attached to the rear frame) we assume no-hands operation. The resulting non-holonomic mechanical model has three velocity degrees of freedom: forward speed $v$, lean rate $\dot{\phi}$ and steering rate $\dot{\delta}$. For the validation we consider the linearized equations of motion for small perturbations of the upright steady forward motion (see Appendix). Note that this uncontrolled model can show, after a lateral perturbation, asymptotically stable motion in a certain speed range, despite the fact that the model is energy conservative.

The experimental system [8] consists of an instrumented bicycle without rider, see Fig. 2. Sensors are present for measuring the roll rate, yaw rate, steering angle, and rear wheel rotation. Trainer wheels prevent the complete fall of the bicycle for unstable conditions. Measurements are recorded for the case in which the bicycle, after being manually pushed off, coasts freely on a level surface. From these measured data, eigenvalues are extracted by means of curve fitting. These eigenvalues are then compared with the results from the linearized equations of motion of the model. The literature on experimental verification of modelling aspects in single-track vehicles can be divided into two main sections: bicycles and motorcycles. It is known from experience that tires play a dominant role in the dynamic behaviour of a motorcycle at moderate to high speed. Since the model under study here 


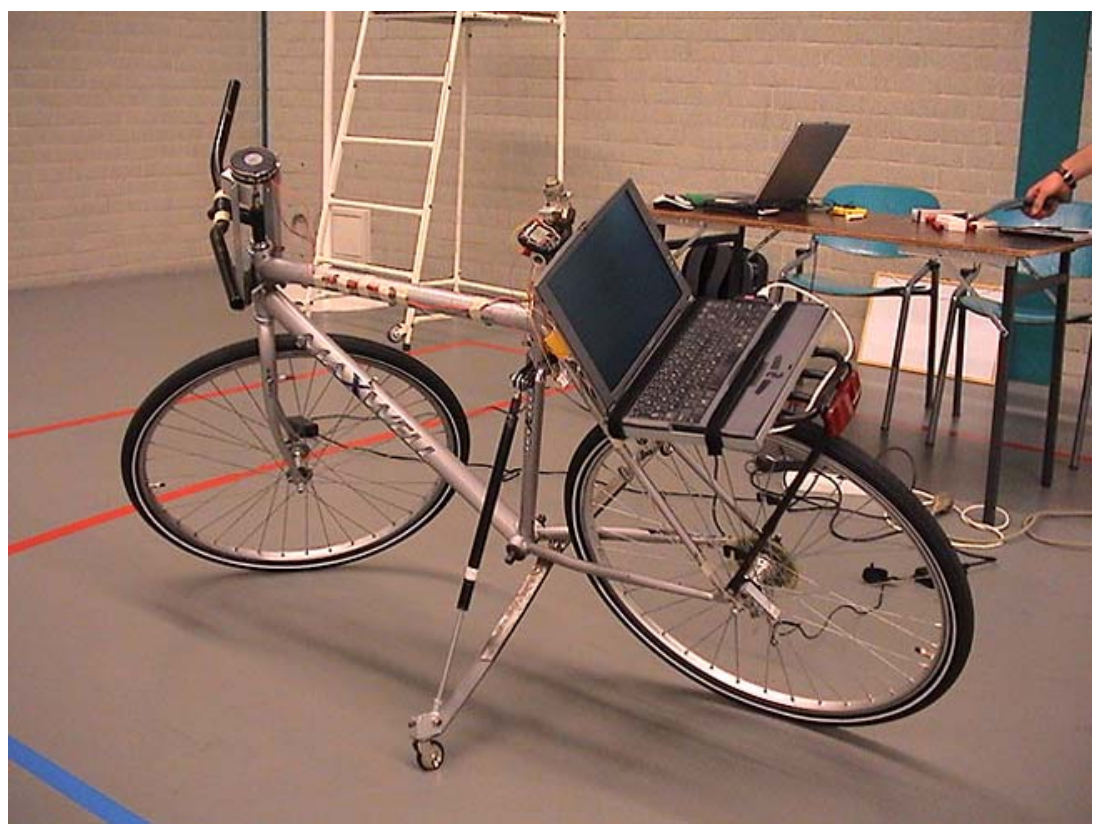

Fig. 2 Instrumented bicycle with all the measurement equipment installed. Sensors are present for measuring the roll rate, yaw rate, steering angle, and rear wheel rotation. Data are collected via a USB-connected data acquisition unit on the laptop computer, mounted on the rear rack

operates at low speed and assumes ideal rolling contact, it seems that many motorcycle studies lie outside the current scope.

Döhring $[4,5]$ was the first to measure the lateral motion of a single-track vehicle. Although he measured on three motorcycles, he compared his results with the same numerical model as presented here. Rice and Roland [11] measured the lateral stability and control of two distinct bicycles, both in hands-free as well as controlled operation. Roland and Lynch [14] performed an extensive study in lateral bicycle dynamics. They measured tire characteristics and then performed a number of tests on an instrumented uncontrolled bicycle and compared the results with their model (Roland and Massing [15], Roland [13]). Wächter [20] and Suhr [18] performed some experimental validation for their bicycle dynamics model. Jackson and Dragovan [7] measured the state of a bicycle ridden hands-free and compared the time history with results from their model.

Many measurements have been made on the lateral dynamics of motorcycles. We discuss here only some distinct contributions. Eaton [6] measured the transient response of a light motorcycle to a lateral disturbance and compared this with his model. Rice [12] performed a number of tests on motorcycles in order to obtain performance characteristics for handling and safety. Weir and Zellner [21] tested transient behaviour of motorcycles in some standard manoeuvres and compared some results with their model. Ruijs and Pacejka [16] built a rider robot to validate their computer model eliminating disturbances originating from the human rider. Rider robots have also been built by the group of Kageyama [10] and the Blue Team [2], a group of Berkeley students engaged in the DARPA challenge. The group led by Cossalter [1,3] over the past decade performed an extensive range of measurements on several aspects of the dynamic behaviour of motorcycles. 
The organization of the paper is as follows. After this introduction, the instrumented bicycle and subsequently the measurement of the bicycle design parameters are described. Then the test procedure and the comparison of the experimental and numerical results are presented and discussed. The paper ends with some conclusions.

\section{Instrumented bicycle}

The experimental system consists of an instrumented bicycle without rider. Sensors are present for measuring the roll rate, the yaw rate, the steering angle and the rear wheel rotation. The data are collected on a laptop computer mounted on the rear rack. Trainer wheels prevent the complete fall of the bicycle for unstable conditions. Measurements are recorded for the case in which the bicycle coasts freely on a level surface.

\subsection{The bicycle}

The instrumented bicycle used in the tests was a standard $21^{\prime \prime}$ city-bicycle with $28^{\prime \prime}$ wheels, brand 'Maxwell', type 'Silvercity', see Fig. 2. For the experiments all the superfluous parts on the bicycle were removed. What remained was the rear frame with bottom bracket and the rear rack, the front fork and the handlebar, front wheel and the rear wheel with sprocketwheel cassette.

Sidewheels were added to the bicycle to reduce the maximum lean angle by which the bicycle could tip over to about $30^{\circ}$. The sidewheels were connected via a suspension construction consisting of two metal strips acting as leaf springs and two telescopic dampers, which had the air removed, to act as a guide. The front fork was flipped (rotated by $180^{\circ}$ ) to increase the trail. This lowers the weave speed and by such increases the stable speed range. In this way we were able to make better use of the limited space available and maximal attainable launch speed (running along the bicycle) for the tests. The head tube bearings and the wheel hub bearings were cleaned and reinstalled to reduce play and excessive friction. New standard road tires, $28 \times 13 / 8$ (Halfords brand), and new inner tubes were mounted. The tires were inflated to 3.5 bar. The rims were checked for trueness. No radial or lateral misalignment was noted.

\subsection{Measuring equipment}

The measurement equipment was chosen such that there would be no effect on the dynamic behaviour of the system other than added weight or inertia (no extra degrees of freedom by non-rigidly attached parts). Furthermore, it should be portable from one bicycle to another and had to withstand moderate shocks. Within a limited budget (M.Sc. project) the following equipment was chosen:

Angular rate sensors: Two angular rate gyros, brand Silicon Sensing CRS03 with a rate range of $\pm 100 \mathrm{degs} / \mathrm{s}$ were used to measure the lean rate and yaw rate of the rear frame. The two rate sensors were placed perpendicular to each other on a $T$ profile that was clamped to the saddle post, as in Fig. 3a. A rotational spirit level was placed on top of the yaw rate sensor to ensure that it was placed horizontally.

Steering angle sensor: A potentiometer (Duncan Electronics Inc. 1801 Single turn, 358 degree) was used with the following specifications: Resistance: $20 \mathrm{k} \Omega \pm 3 \%$ and Linearity: $\pm 0.75 \%$. Most "standard" Dutch bicycles have a head tube with an outer diameter of 


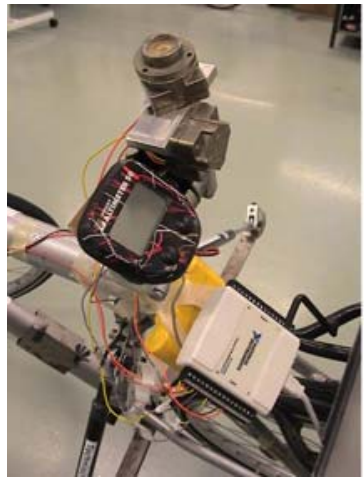

(a)

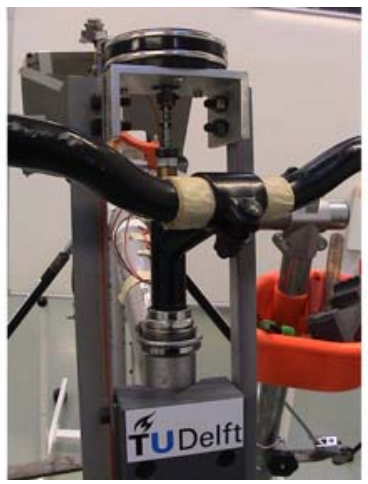

(b)

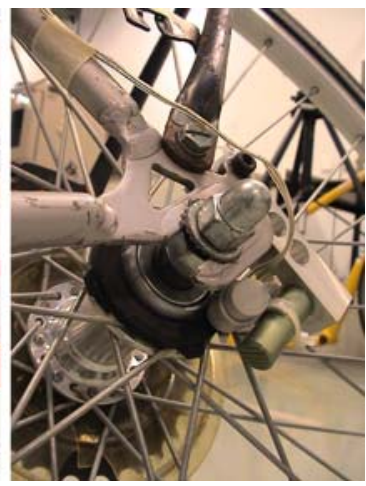

(c)

Fig. 3 Close-ups of the mounted instruments of the instrumented bicycle from Fig. 2: a two angular rate sensors, spirit level, speedometer display, battery pack and USB data acquisition unit, $\mathbf{b}$ steering angle potentiometer, $\mathbf{c}$ forward speed sensor which consists of a 10 magnet ring (black) and pick-up

$34 \mathrm{~mm}$. Therefore, a bracket with two vertically mounted steel L shaped rods connected to an aluminium U-profile kept the potentiometer in position, as in Fig. 3b. The rods also restricted the maximum angle that the front frame could make with respect to the rear to about $30^{\circ}$, reducing the chance that the bicycle could flip over. Minimal play was achieved between the potentiometer and the front frame by directly connecting the sensor to the steering axis via a flexible coupling that compensated for a small misalignment of the steering axis with the sensor axis. A transmission was thus avoided. A drawback to this method was that the output range of the signal was small.

Forward speed sensor: Forward speed was measured by means of a standard speedometer (Avocet Altimeter 50). This unit uses a magnetic ring with 10 north/south poles and a pick-up. The magnetic ring was mounted on the rear wheel (forward speed is defined as $\left.v=-\dot{\theta}_{r} R_{r w}\right)$ and the pick-up was connected to one of the analogue channels of the USB data acquisition unit, as in Fig. 3c. The speedometer was placed on an aluminium shaft that was connected to the saddle clamp. With the speedometer in this position the speed values shown on its screen were clearly legible when running behind and alongside the bicycle. However, the refresh rate of the computer was too slow and full attention of the launcher was usually needed to launch the bicycle in a proper manner.

Data acquisition unit: A Universal Standard Bus (USB) connected data acquisition unit (National Instruments NI-USB-6009) was used. This unit has eight 14-bit analogue input channels, 12 digital I/O lines, two analogue outputs and one counter. Only the analogue inputs were used. The data acquisition unit was bolted to the rear carrier as in Fig. 3a.

Laptop: For data collection a laptop was used (ACER 340 Travelmate with a Sitecom USB 2.0 PCMCIA card installed). The laptop was placed on the rear carrier, reducing the dynamic stability of the bicycle [14], but the fixation was easily achieved whilst remaining compatible for other bicycles. The laptop was placed in a steel frame with padding that was mechanically bolted to the rear carrier such that the centre of mass of the laptop and steel frame was over the bicycle symmetry plane. The laptop was kept in the frame by two Velcro straps.

Battery pack: The DC power supply for the rate gyros was a battery pack (BMI NI-CD $4.8 \mathrm{~V} 2100 \mathrm{mAh}$ ) cable-tied to the rear frame as in Fig. 3a. 
Table 1 Design parameters for the bicycle model from Fig. 1 together with measured values for the instrumented bicycle from Fig. 2

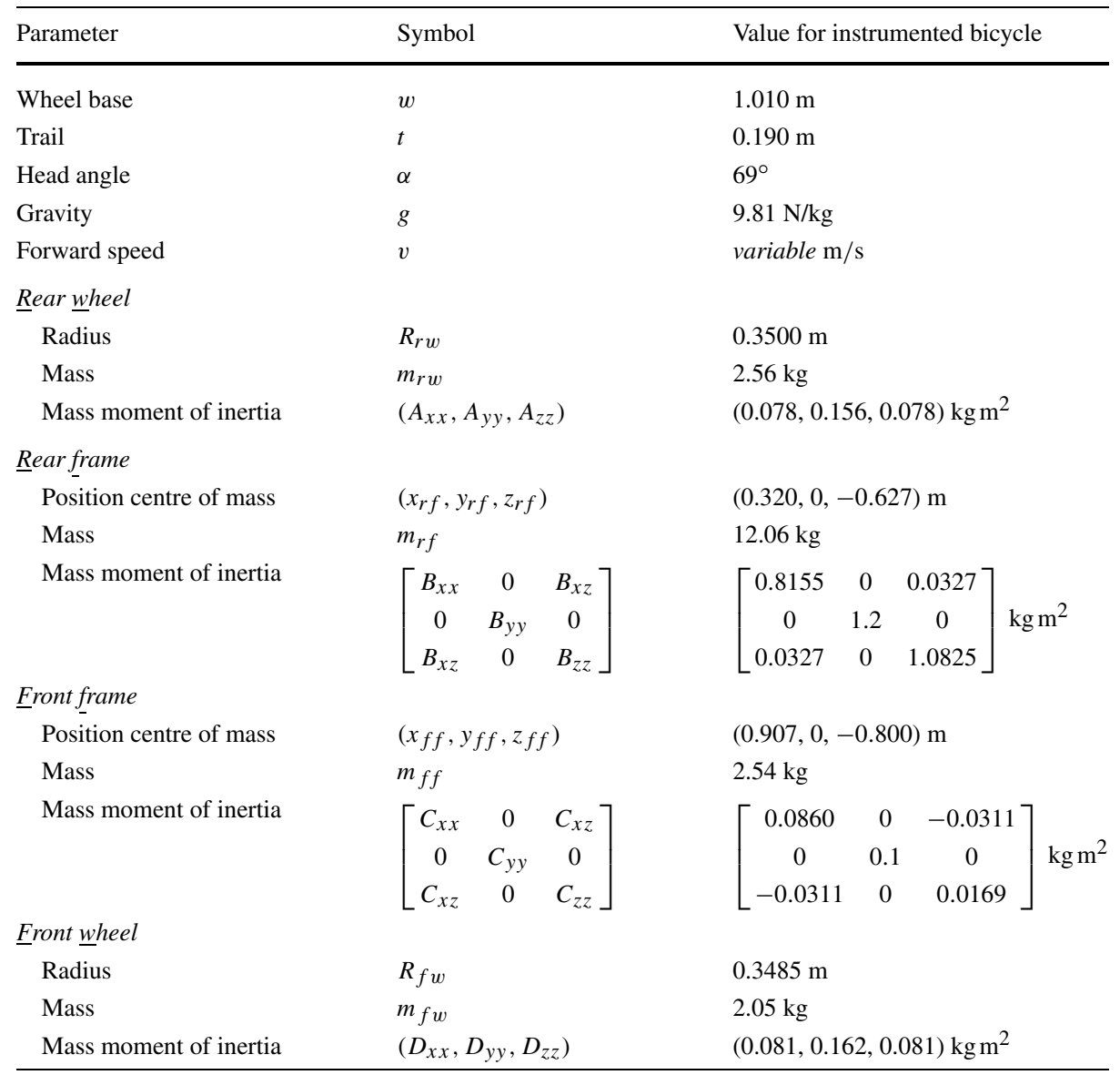

Subscripts used are: $r w$ for the rear wheel, $r f$ for the rear frame, $f f$ for the front frame, $f w$ for the front wheel, $x, y$, and $z$ are the directions along the global $x y z$-axes with the origin $O$ at the rear contact point. Centre of mass locations for the individual parts are expressed with respect to the origin $O$, whereas mass moments of inertia are about the centres of mass and use axes that are initially aligned with the global $x y z$ axes

\section{Measuring the bicycle design parameters}

In order to predict the dynamic behaviour of the instrumented bicycle by means of the linearized equations of motion of the bicycle model (see Appendix), all 25 bicycle design parameters had to be measured. These measured values are presented in Table 1. The bicycle was measured with all the measurement equipment installed, including the laptop and sidewheels. The bicycle parameters can be divided into two groups, geometrical properties and mass properties.

\subsection{Geometrical properties}

The geometrical parameters shown schematically in Fig. 1 were measured as follows. 
Wheelbase: The distance from the centre of the front contact patch to the centre of the rear contact patch measured on level ground whilst the front fork was aligned with the rear frame.

Head angle: Measured on level ground with the bicycle vertical by placing a protractor with integrated spirit level against the front of the head tube.

Trail: On level ground with the bicycle vertical and with the front fork facing forwards. By extending the head tube by means of a plate and rod. The trail was then determined by measuring the distance parallel to the $x$-axis of the bicycle between the contact point of the front wheel and the intersection of the head tube with the ground.

Wheel radius: This was determined by measuring the distance covered by the bicycle, when the wheel that was being measured travelled nine full rotations along a straight line on a level floor, where the tires were inflated to 3.5 bar. Nine rotations were used as this was the maximum amount that could be measured with a $30 \mathrm{~m}$ long measuring tape. The measured distance was then divided by $18 \pi$ to get the effective rolling radius.

\subsection{Mass properties}

The numerical model requires the mass and inertia properties of the four separate parts: rear wheel, rear frame assembly, front handlebar assembly, and front wheel. For each part the mass, the location of its centre of mass and the mass moments of inertia with respect to axes through the centre of mass and parallel to the global $x y z$-axes in the reference position (see Fig. 1) had to be found.

The mass of the parts was easily measured on a set of scales to an accuracy of $\pm 0.01 \mathrm{~kg}$. Determining the mass moments of inertia of the parts turned out to be more difficult.

Given the simple geometry of the four bicycle parts, one would think it would be easy to calculate the mass moments of inertia from a measured geometry model. However, serious problems were encountered:

1. All the measuring equipment, their brackets and the sidewheel construction had to be modelled. Since none of the sensors or the computer have constant densities, assigning the correct mass moments of inertia to them without actually measuring them is almost impossible.

2. The wall thickness of most of the tubes was not known. Thus without drilling a hole in most of the tubes these could not be measured. It was also unknown if the wall thickness was constant in each tube.

3. Most of the tubes of the front and rear frame do not have a constant cross-section, increasing the complexity of the model.

Thus it was decided that the mass moment of inertia parameters of the instrumented bicycle would be measured instead. To this end, a torsional pendulum was constructed.

\subsubsection{Measuring the mass moments of inertia}

The mass moments of inertia of the four individual parts were measured by means of a torsional pendulum, as shown in Fig. 4. The torsional pendulum was made of a slender steel rod, $1 \mathrm{~m}$ long and $5 \mathrm{~mm}$ diameter, that was clamped vertically at the upper end. The lower end of the rod was connected by a stiff coupling to the bicycle part to be measured. The torsion rod was clamped over a length of $4 \mathrm{~cm}$ at both ends leaving a free length of $92 \mathrm{~cm}$.

The bicycle part was clamped such that its centre of mass was aligned with the rotational axis of the rod. This ensured that measurement was made about a centre of mass axis of 
Fig. 4 Torsional pendulum with rear frame clamped in the bracket. The centre of mass of the rear frame is aligned with the vertical axis of the torsional rod

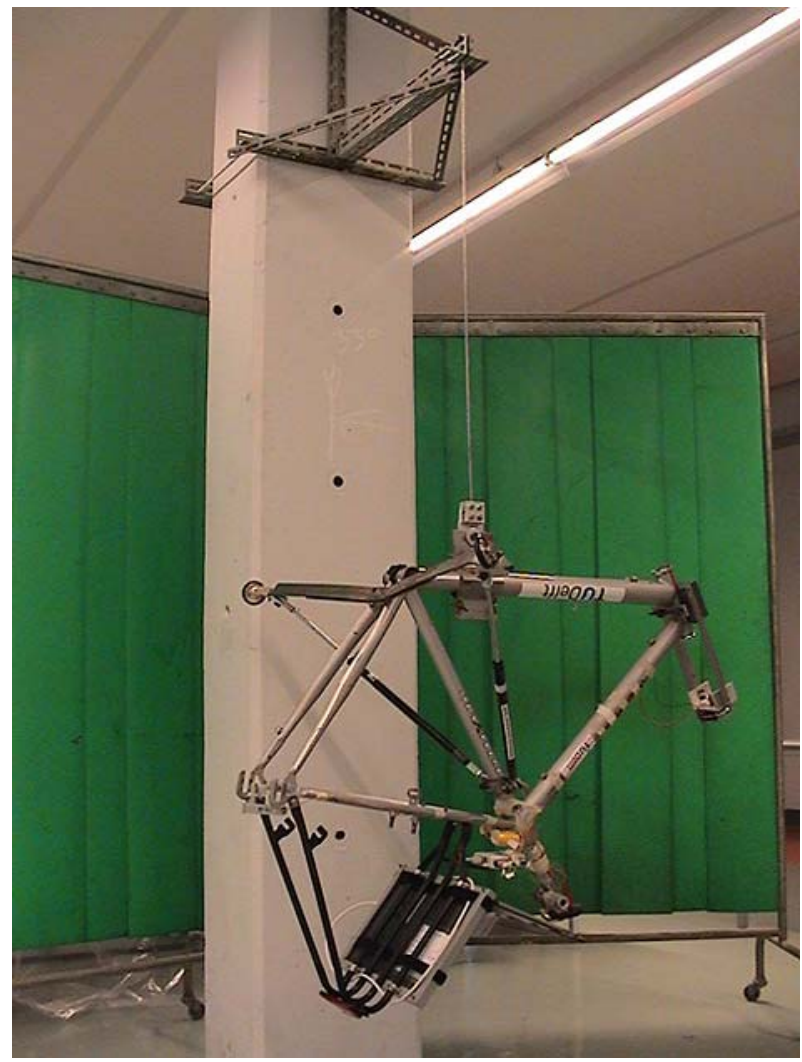

the part and that there was no bending moment in the rod that could disturb the angular oscillation of the system.

The bicycle part was then manually set into an angular oscillation about the vertical axis and the time period of the oscillation was measured. The part was given only a small initial rotation to ensure elastic material behaviour in the rod.

The equation of motion for the torsional pendulum is given by

$$
I_{M} \ddot{\theta}+\left[\frac{G I_{P}}{l}\right] \theta=0
$$

where $I_{M}$ is the mass moment of inertia of the part about the vertical axis, $\ddot{\theta}$ the angular acceleration of the lower end of the rod, $l$ is the length of the rod, $G$ the shear modulus of elasticity of the rod material (steel was used) and $I_{P}$ the polar area moment of inertia of the cross-section of the rod. The mass moment of inertia of the clamp that held the bicycle part was very small compared with that of the bicycle part, and its contribution was neglected. Therefore the mass moment of inertia of the measured part along the vertical axis could be calculated as

$$
I_{M}=\left(\frac{T}{2 \pi}\right)^{2}\left[\frac{G I_{P}}{l}\right],
$$


where $T$ is the time period for one oscillation. Actually, the torsional stiffness $G I_{P} / l$ was not calculated but measured by a torsional vibration experiment with a known object (a horizontal slender rod was used), which ensured more accurate results.

Under the assumption that all parts have a vertical symmetry plane $(x z)$ the mass moment of inertia matrix expressed in the body fixed $x y z$-axes at the centre of mass takes the following form

$$
\mathbf{I}=\left[\begin{array}{ccc}
I_{x x} & 0 & I_{x z} \\
0 & I_{y y} & 0 \\
I_{x z} & 0 & I_{z z}
\end{array}\right] .
$$

Therefore only four out of the six mass moment of inertia terms of the $3 \times 3$ symmetric inertia matrix had to be determined. Moreover, since the pitch motion is not present in the linearized model of the upright bicycle there was no need to measure the mass moment of inertia along this pitch axis, $I_{y y}$, and an educated guess was made. This reduced the necessary number of measurements to three.

For the rear frame assembly (all measurement equipment installed) the experiment was carried out on the three main frame tubes of the rear frame: the sloping lower tube, the top tube and the seat tube. For the front frame the experiment was carried out with the clamp on the head tube about the pitch and roll axes and about the handlebar for the yaw axis. Figure 4 shows the rear frame, with the saddle tube held in the clamp.

The measurement of the wheel inertia was done in two separate tests. The first was carried out using the torsional pendulum to measure the mass moment of inertia about the wheel's global $x$-and $z$-axis (identical). The second test was to determine the mass moment of inertia about the rotation axis, $I_{y y}$. In a compound pendulum experiment the wheel was hung from a horizontally placed nail and given a small offset to bring the wheel into an oscillation and the time was measured. Then, with the known total mass and distance to the centre of mass, the mass moment of inertia about the axle axis could be calculated using the parallel axis theorem. Note that in this way the mass moment of inertia was overestimated a little, because of the small non-rotating parts at the hub.

The wheels were weighed as one complete unit even though in the linearized dynamic model the wheels are modelled as only the rotating part of the wheel. The inaccuracy for the model was considered negligible because the masses of the non-rotating parts at the hub are small.

\subsection{Processing of the mass moments of inertia}

In order to be able to calculate the mass moments of inertia in the global axes about the centre of mass for the different parts, the mass moment of inertia tensors had to be rotated. To do this the angle under which the frame had been hung had to be calculated. Photographs were taken with the camera held horizontally, perpendicular to the frame. Each photograph was taken in the same position and the same distance from the part for each of the experiments. Then together with the information that the top tube has a $5^{\circ}$ slope relative to the global $x$-axis when the bicycle is on level ground, the angle under which the frame was hung in each case relative to the global $x$-axis was calculated.

Next the position of the centre of mass was found. Using the same photographs and placing them on top of one another and extending the torsion rods the location of the centre of mass was found. The location of the centre of mass of the part as found in the photograph was then transformed to the global coordinates by measuring tube lengths in the photographs, comparing them with the actual lengths and calculating the scale factor. 
For the rear frame assembly, three measurements, $I_{i}$, about three different axes, $\mathbf{e}_{i}$, in the $x z$-plane were made. The three unknown terms, $I_{x x}, I_{x z}$, and $I_{z z}$, from the mass moment of inertia matrix (3) were then solved from the set of linear equations

$$
\mathbf{e}_{i}^{T} \mathbf{I e}_{i}=I_{i}, \quad i=1,2,3 .
$$

The front fork and handlebar assembly was measured in the same manner, where it should be noted that the mass moment of inertia about the steering axis was small in comparison with the other entries in the inertia matrix and should have been measured with a more compliant torsion bar.

\subsection{Measured design parameters and linearized equations of motion}

The measured mass moments of inertia and centres of mass of all parts together with the other measured bicycle parameters are presented in Table 1. From these measured parameters the coefficient matrices of the linearized equations of motion from the Appendix, (31-34), are calculated as:

$$
\begin{aligned}
& \mathbf{M}=\left[\begin{array}{l}
7.98981,0.89569 \\
0.89569,0.29857
\end{array}\right], \quad \quad \mathbf{C}_{1}=\left[\begin{array}{cc}
0, & 7.17025 \\
-0.59389, & 1.32610
\end{array}\right], \\
& \mathbf{K}_{\mathbf{0}}=\left[\begin{array}{cc}
-109.91168,-13.45745 \\
-13.45745, & -4.82272
\end{array}\right], \quad \mathbf{K}_{2}=\left[\begin{array}{cc}
0,11.19798 \\
0,1.42200
\end{array}\right] \text {. }
\end{aligned}
$$

Then, with these coefficient matrices the characteristic equation,

$$
\operatorname{det}\left(\mathbf{M} \lambda^{2}+v \mathbf{C}_{\mathbf{1}} \lambda+\mathbf{K}_{\mathbf{0}}+v^{2} \mathbf{K}_{\mathbf{2}}\right)=0
$$

can be formed and the eigenvalues, $\lambda$, can be calculated. These calculated eigenvalues, in the forward speed range of $0 \leq v \leq 9 \mathrm{~m} / \mathrm{s}$, are presented by the continuous lines in Fig. 7. In principle there are up to four eigenmodes, where oscillatory eigenmodes come in pairs. Two are significant and are traditionally called the capsize mode and weave mode. The capsize mode corresponds to a real eigenvalue with eigenvector dominated by lean: when unstable, the bicycle just falls over like a capsizing ship. The weave mode is an oscillatory motion in which the bicycle sways about the headed direction. The third remaining eigenmode is the overall stable castering mode which corresponds to a large negative real eigenvalue with eigenvector dominated by steering.

\section{Experimental procedure and results}

Measurements were recorded for the case in which the bicycle coasted freely on a level surface. From these measured data eigenvalues were extracted by means of curve fitting. These eigenvalues were then compared with the results from the linearized equations of motion of the model. As a result, the model appeared to be fairly accurate for the low-speed low-frequency behaviour. 


\subsection{Expected motions}

Looking at the eigenvalue plot in Fig. 7, the following bicycle motions during the experiments can be expected. At low speed the motion of the free-coasting laterally-perturbed bicycle will be dominated by the unstable weave motion. Both the capsize and the castering mode are very stable here and any initial transient will quickly die out. The time frame for measurement will be short due to the unstable nature of the weave motion. Then in the stable speed range, again the motion will be dominated by the oscillatory weave motion. The moderately stable/unstable capsize motion will only give a small offset in the lean rate. Here, the measurement window will be large since the motion is oscillatory stable.

\subsection{Test procedure}

The experiments were carried out in the university sports hall. The $42 \times 25 \mathrm{~m}$ sports hall had a dry, level floor with a rubbery surface layer as can be found in any modern sports hall.

A total of 76 runs were carried out. Each run consisted of three segments. In the first segment the bicycle was brought up to speed and during high speed runs the bicycle was then laterally perturbed by hitting the rear frame. In the second segment the bicycle was allowed to coast freely and in third segment the bicycle was brought back to a standstill.

A restriction on the spread of the measurements was the top speed of the person propelling the bicycle along as the person not only had to be able to accelerate the bicycle in as short a distance as possible but also had to be able to run alongside the bicycle and bring it back to a standstill before it collided with the wall.

To measure the dynamic response of the bicycle at the different speeds and to calculate the corresponding motion eigenvalues the bicycle had to show some lateral dynamics. At speeds below the stable speed range no external excitation was required. Due to small imperfect or non-perfect initial conditions, the bicycle always started to weave about its general heading and this motion was measured. Moreover, the small asymmetry caused the bicycle to lean to the right when released, which thereby ended up rolling in a large circle rather than a straight line.

For runs in the stable speed range, the bicycle set itself in an upright position and showed no dynamic behaviour unless it was given a lateral excitation. This excitation was accomplished by applying a lateral impulse to the bicycle by simply hitting the bicycle's rear frame by hand in the lateral direction at approximately the insertion of the saddle pillar with the down tube.

Two runs were carried out to determine the zero speed inverted pendulum eigenvalue. Eight runs were carried out at low speed (around $2 \mathrm{~m} / \mathrm{s}$ ), and the majority of the tests (56) were carried out in the transition region from unstable weave to stable weave motion (3.5$5 \mathrm{~m} / \mathrm{s}$ ). Ten tests were carried out at higher speeds up to about $6 \mathrm{~m} / \mathrm{s}$. At forward speeds of less than $2 \mathrm{~m} / \mathrm{s}$ the bicycle was very unstable, giving only a very short time window for measurements.

\subsection{Stored data}

The frequency of the weave of motion is low, at the order of $1 \mathrm{~Hz}$ (see Fig. 7) and therefore only a low sample rate was needed here. However, the measurement of the forward speed by means of the 10-magnet ring needed a higher sampling rate. The first tests were measured with a $100 \mathrm{~Hz}$ sample frequency. Later the sample frequency was set to $400 \mathrm{~Hz}$ to ensure 
no aliasing in the speed signal would take place when testing at a higher speed. Unfortunately, higher sampling frequencies gave a very erratic signal at the recorder steering angle potentiometer signal. The recorded data for each run were stored in a text file.

Every run was recorded on video, too. An example of such a recording can be found at [19]. This turned out to be essential for the processing of the run data and helped to identify nonstandard measurements, the quality of the launch, etc. It was thus possible to compare the recorded data afterwards with the video images and to extract the relevant data for the calculation of the eigenvalues from each file.

\subsection{Data analysis}

For each run the raw data were transferred to Matlab and first inspected visually. A plot of the raw data for run 52 is shown in Fig. 5. In the figure the signals from top to bottom are: battery voltage, steering angle, lean rate, yaw rate, and speedometer. These graphs were used together with the videos of the runs to locate the time window in which the bicycle coasted freely for each run. Once this window was manually located, a curve fit of the time histories of the eigenmotions was performed on the lean rate data to extract the measured eigenvalues as shown in Fig. 6. Above a forward speed of $0.1 \mathrm{~m} / \mathrm{s}$ there is in principle a sum of three eigenmodes to be fitted: the castering mode, the capsize mode and the oscillatory weave mode, as shown in Fig. 7. The castering mode is highly damped and will vanish quickly from the transient signal. The capsize mode is also reasonably damped below the weave speed and is mildly unstable above the weave speed, resulting here in a small and slow lean rate offset. Therefore only an exponentially damped or growing oscillatory weave motion

\section{signals [V]}

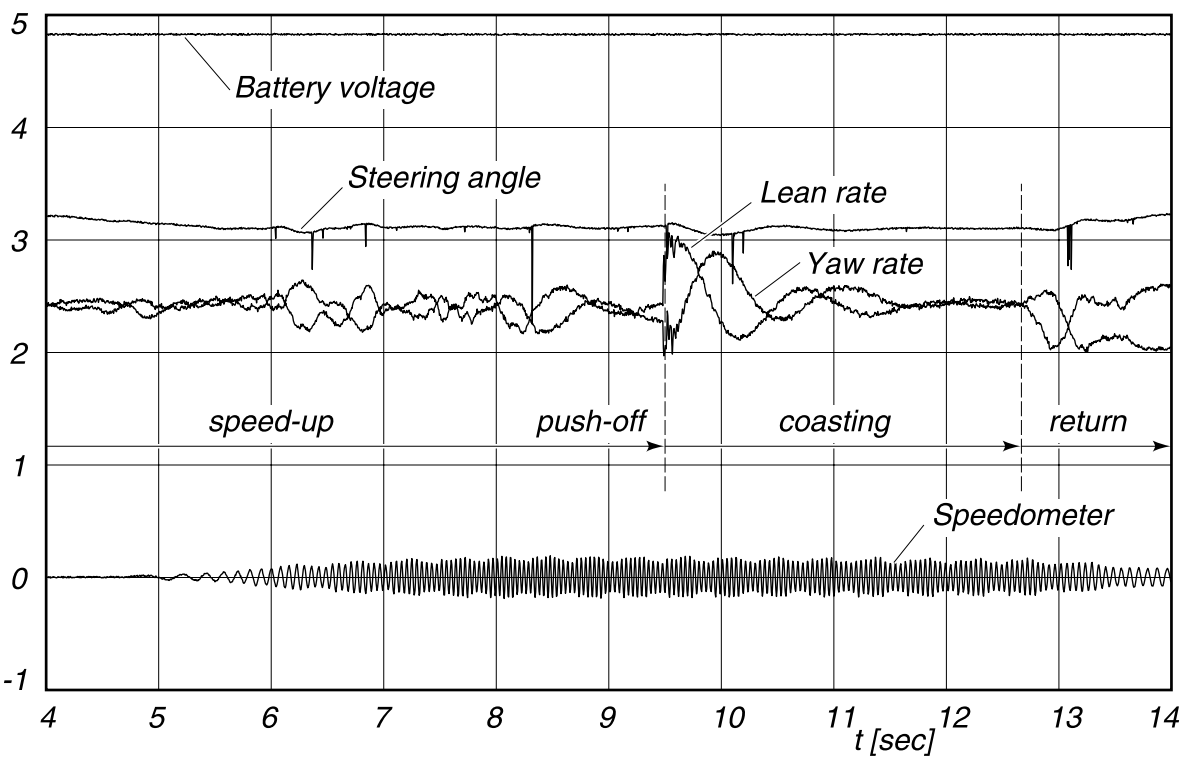

Fig. 5 The raw measured data from run 52. The signals from top to bottom are: battery voltage, steering angle, lean rate, yaw rate, and speedometer. The forward speed is around $5 \mathrm{~m} / \mathrm{s}$, which is clearly within the stable speed range (see Fig. 7). Note the three different motion regimes: speed-up, coasting, and return to base 


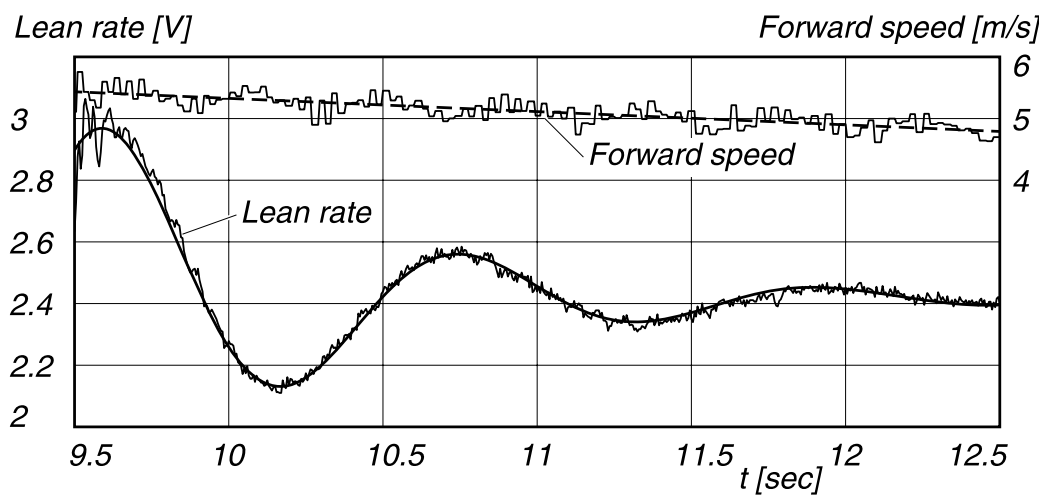

Fig. 6 Least-squares curve fit of the oscillatory stable lean rate time history (solid smooth curve) to the measured lean rate (ragged line) for run 52, together with the measured forward speed (staircase line) and linear regression of the forward speed (dashed line). Note the slight decrease in forward speed (from 5.4 to 4.8 $\mathrm{m} / \mathrm{s}$ ) during the measurement. The extracted weave eigenvalue within the time window of $9.9 \leq t \leq 12.5 \mathrm{~s}$ is $\lambda_{\text {weave }}=-1.16 \pm 5.44 \mathrm{i} 1 / \mathrm{s}$

was fitted to the data. The function to be fitted on the measured lean rate was taken as

$$
\dot{\phi}=c_{1}+e^{d t}\left[c_{2} \cos (\omega t)+c_{3} \sin (\omega t)\right] \text {, }
$$

with the weave frequency $\omega=\operatorname{Im}\left(\lambda_{\text {weave }}\right)$, the weave damping $d=\operatorname{Re}\left(\lambda_{\text {weave }}\right)$ and the three constants $c_{1}$ for the offset, $c_{2}$ for the cosine amplitude, and $c_{3}$ for the sine amplitude. Since the weave frequency and damping appear in a non-linear way in the function, a non-linear least-squares fitting method was used (Matlab's fminsearch) to extract the eigenvalues.

The speedometer signal (see Fig. 5) was an oscillatory signal with a frequency of ten times the rear wheel rotation frequency. The signal was converted to a forward speed by counting the time between successive zero crossings. As each crossing represents a $1 / 20$ th of a complete rear wheel rotation, an average speed for that portion could be calculated; this is the staircase line in Fig. 6. As the forward speed during the coasting section of the measurements slowly decreased due to air resistance and rolling resistance, a speed range was assigned to the calculated $\lambda$ 's instead of one specific speed. This speed range was calculated by looking at the linear regression of the speed for the chosen time window, see Fig. 6 .

At zero speed two measurement were made. Since the bicycle just falls over in an inverted pendulum manner, only an exponential growing solution was fitted to the lean rate data and only one eigenvalue was extracted. Finally, in Fig. 7 the measured eigenvalues for all runs were plotted on top of the calculated eigenvalues, with horizontal bars used to indicate the forward speed variation during the measurements.

\subsection{Discussion}

At speeds above $3 \mathrm{~m} / \mathrm{s}$, the predicted weave frequency and damping by the model were forecasted accurately. The transition from the unstable to the stable region around the weave speed is accurately described by the model.

In the unstable speed region, below $3 \mathrm{~m} / \mathrm{s}$, it turned out to be very difficult to measure the motion of the bicycle. The time window for measurement was very short compared with the time period of the weave motion. Therefore, trying to fit only a part of a harmonic function 


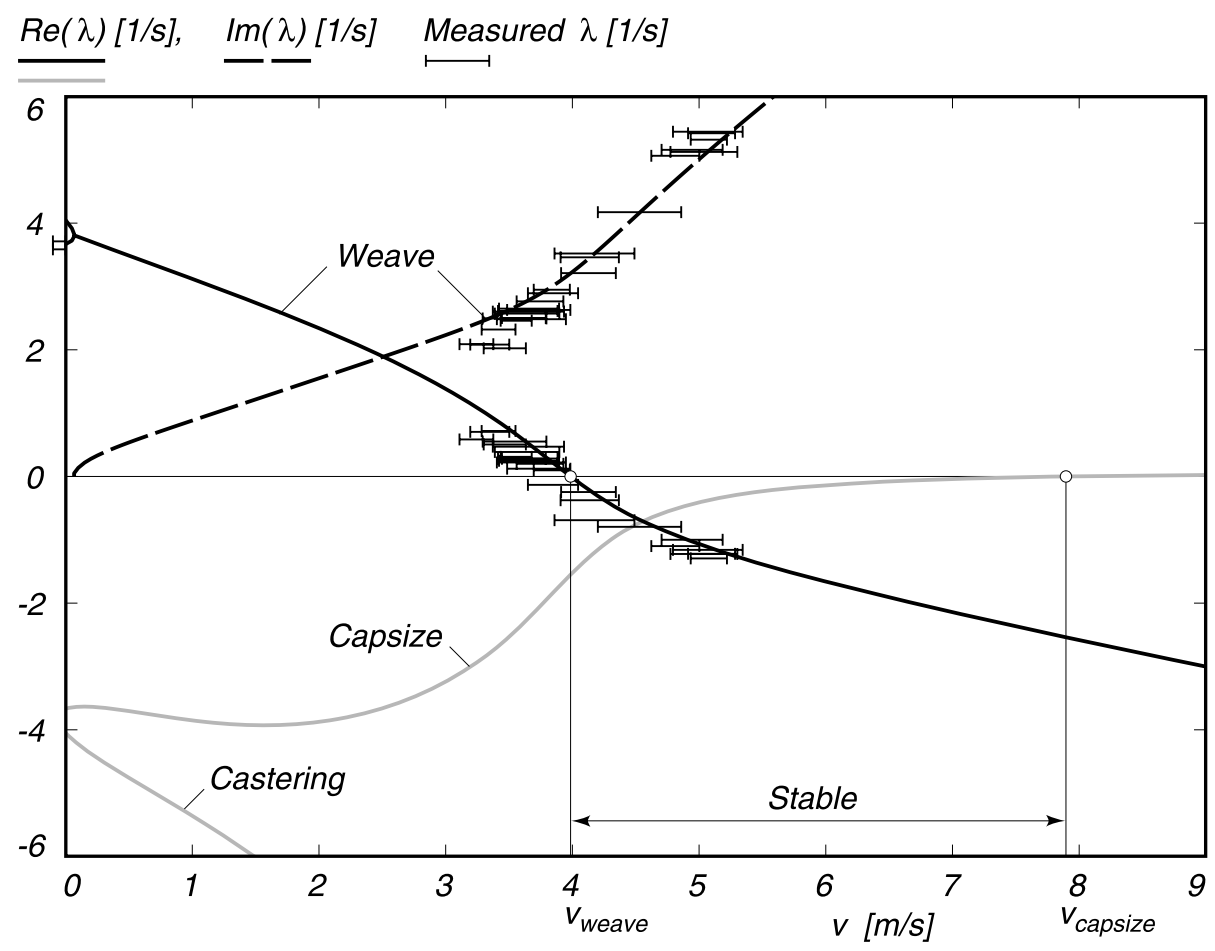

Fig. 7 Measured eigenvalues $\lambda$ (horizontal bars) and calculated eigenvalues $\lambda$ (continuous lines) for the instrumented bicycle from Figs. 1 and 2, and Table 1, in the forward speed range of $0 \leq v \leq 9 \mathrm{~m} / \mathrm{s}$. For the measured values only the weave motion is considered. The lengths of the horizontal bars indicate the forward speed range during the measurement. For the calculated values the solid lines correspond to the real part of the eigenvalues and the dashed line corresponds to the imaginary part of the eigenvalues. The zero crossings of the real part of the eigenvalues are for the weave motion at the weave speed $v_{\text {weave }} \approx 4.0 \mathrm{~m} / \mathrm{s}$ and for the capsize motion at capsize speed $v_{\text {capsize }} \approx 7.9 \mathrm{~m} / \mathrm{s}$. The speed range for the asymptotic stability of the instrumented bicycle is $v_{\text {weave }}<v<v_{\text {capsize }}$

to the measured data turned out to be very difficult and the results showed considerable spread.

The yaw rate signal was of the same quality as the lean rate signal, but the steering angle signal turned out to be too small and too erratic to use.

\section{Conclusions}

The experimental results show a fairly good agreement with the results obtained by a linearized analysis on a three-degree-of-freedom dynamic model of an uncontrolled bicycle. This shows that the tire compliance, tire slip, and frame and fork compliance are not important for the lateral dynamics of the bicycle in the forward speed range of 0 to $6 \mathrm{~m} / \mathrm{s}$. The same result was found from theoretical considerations by Limebeer and Sharp [9], which showed that eigenfrequencies and damping ratios in the low-speed range were only slightly influenced by including these effects. For a bicycle with a rider and a normal trail, the relative movements of the rider and the tire properties might prove to have more influence. 
The validated bicycle model can be used, among others, to design a controller for the (mostly) unstable bicycle.

\section{Appendix: Linearized equations of motion}

This appendix gives a summary of the algorithmic interpretation of the linearized equations of motion as presented in [17].

Consider the bicycle from Fig. 1 and Table 1. The linearized equations of motion expressed in terms of small changes in the lateral degrees of freedom being the rear frame roll angle, $\phi$, and the steering angle, $\delta$, from the upright straight ahead configuration $(\phi, \delta)=(0,0)$, at a forward speed of $v=-\dot{\theta}_{r} R_{r w}$ have the form

$$
\mathbf{M} \ddot{\mathbf{q}}+v \mathbf{C}_{\mathbf{1}} \dot{\mathbf{q}}+\left[\mathbf{K}_{\mathbf{0}}+v^{2} \mathbf{K}_{\mathbf{2}}\right] \mathbf{q}=\mathbf{f},
$$

where the time-varying variables are $\mathbf{q}=[\phi, \delta]^{T}$ and $\mathbf{f}=\left[T_{\phi}, T_{\delta}\right]^{T}$. The coefficients in this equation are: a constant symmetric mass matrix, $\mathbf{M}$, a damping-like (there is no real damping) matrix, $v \mathbf{C}_{\mathbf{1}}$, which is linear in the forward speed $v$, and a stiffness matrix which is the sum of a constant symmetric part, $\mathbf{K}_{\mathbf{0}}$, and a part, $v^{2} \mathbf{K}_{\mathbf{2}}$, which is quadratic in the forward speed. The forces on the right-hand side, $\mathbf{f}$, are the applied forces which are energetically dual to the degrees of freedom q. For the bicycle model the first is $T_{\phi}$, the action-reaction roll torque between the fixed space and the rear frame. The second force is $T_{\delta}$, the actionreaction steering torque between the rear frame and the front frame about the steering axis. In the case of an uncontrolled bicycle, both of these torques are taken to be zero.

The entries in the constant coefficient matrices $\mathbf{M}, \mathbf{C}_{\mathbf{1}}, \mathbf{K}_{\mathbf{0}}$, and $\mathbf{K}_{\mathbf{2}}$ are defined algorithmically as follows. For the system as a whole, calculate the total mass and the corresponding centre of mass with respect to the origin $O$ as

$$
\begin{aligned}
m_{t} & =m_{r w}+m_{r f}+m_{f f}+m_{f w}, \\
x_{t} & =\left(x_{r f} m_{r f}+x_{f f} m_{f f}+w m_{f w}\right) / m_{t}, \\
z_{t} & =\left(-R_{r w} m_{r w}+z_{r f} m_{r f}+z_{f f} m_{f f}-R_{f w} m_{f w}\right) / m_{t} .
\end{aligned}
$$

For the system as a whole, calculate the relevant mass moments and products of inertia at the origin $O$ along the global axes as

$$
\begin{aligned}
& T_{x x}=A_{x x}+B_{x x}+C_{x x}+D_{x x}+m_{r w} R_{r w}^{2}+m_{r f} z_{r f}^{2}+m_{f f} z_{f f}^{2}+m_{f w} R_{f w}^{2}, \\
& T_{x z}=B_{x z}+C_{x z}-m_{r f} x_{r f} z_{r f}-m_{f f} x_{f f} z_{f f}+m_{f w} w R_{f w}, \\
& T_{z z}=A_{z z}+B_{z z}+C_{z z}+D_{z z}+m_{r f} x_{r f}^{2}+m_{f f} x_{f f}^{2}+m_{f w} w^{2} .
\end{aligned}
$$

Now determine the same properties for the front assembly, comprising the front frame and the front wheel, as

$$
\begin{aligned}
m_{f} & =m_{f f}+m_{f w}, \\
x_{f} & =\left(x_{f f} m_{f f}+w m_{f w}\right) / m_{f}, \\
z_{f} & =\left(z_{f f} m_{f f}-R_{f w} m_{f w}\right) / m_{f},
\end{aligned}
$$


and calculate the relevant mass moments and products of inertia for the front assembly at the centre of mass of the front assembly along the global axes as

$$
\begin{aligned}
F_{x x} & =C_{x x}+D_{x x}+m_{f f}\left(z_{f f}-z_{f}\right)^{2}+m_{f w}\left(R_{f w}+z_{f}\right)^{2}, \\
F_{x z} & =C_{x z}-m_{f f}\left(x_{f f}-x_{f}\right)\left(z_{f f}-z_{f}\right)+m_{f w}\left(w-x_{f}\right)\left(R_{f w}+z_{f}\right), \\
F_{z z} & =C_{z z}+D_{z z}+m_{f f}\left(x_{f f}-x_{f}\right)^{2}+m_{f w}\left(w-x_{f}\right)^{2} .
\end{aligned}
$$

Let $\lambda$ be the angle of the steering axis $\lambda=(\sin (\lambda), 0, \cos (\lambda))^{T}$ with the global $z$-axis in the vertical plane,

$$
\lambda=\pi / 2-\alpha
$$

Calculate the perpendicular distance that the centre of mass of the front assembly is ahead of the steering axis,

$$
u=\left(x_{f}-w-t\right) \cos (\lambda)-z_{f} \sin (\lambda) .
$$

Calculate for the front assembly the relevant mass moments and products of inertia along the steering axis and the global axes at points where they intersect as

$$
\begin{aligned}
& F_{\lambda \lambda}=m_{f} u^{2}+F_{x x} \sin (\lambda)^{2}+2 F_{x z} \sin (\lambda) \cos (\lambda)+F_{z z} \cos (\lambda)^{2}, \\
& F_{\lambda x}=-m_{f} u z_{f}+F_{x x} \sin (\lambda)+F_{x z} \cos (\lambda), \\
& F_{\lambda z}=m_{f} u x_{f}+F_{x z} \sin (\lambda)+F_{z z} \cos (\lambda) .
\end{aligned}
$$

Define the ratio of the mechanical trail (i.e., the perpendicular distance that the front wheel contact point is behind the steering axis) to the wheelbase as

$$
f=t \cos (\lambda) / w
$$

Calculate for the rear and the front wheel the angular momentum along the $y$-axis divided by the forward speed, together with their sum as

$$
\begin{aligned}
& S_{r}=A_{y y} / R_{r w}, \\
& S_{f}=D_{y y} / R_{f w}, \\
& S_{t}=S_{r}+S_{f} .
\end{aligned}
$$

Define a frequently appearing static moment term as

$$
S_{u}=m_{f} u+f m_{t} x_{t} .
$$

The elements of the symmetric mass matrix are

$$
\begin{aligned}
& M(1,1)=T_{x x}, \\
& M(1,2)=F_{\lambda x}+f T_{x z}, \\
& M(2,1)=M(1,2), \\
& M(2,2)=F_{\lambda \lambda}+2 f F_{\lambda z}+f^{2} T_{z z} .
\end{aligned}
$$


The "damping" matrix which is to be multiplied by the forward speed, $v$, is given by

$$
\begin{aligned}
& C_{1}(1,1)=0, \\
& C_{1}(1,2)=f S_{t}+S_{f} \cos (\lambda)+T_{x z} \cos (\lambda) / w-f m_{t} z_{t}, \\
& C_{1}(2,1)=-\left(f S_{t}+S_{f} \cos (\lambda)\right), \\
& C_{1}(2,2)=F_{\lambda z} \cos (\lambda) / w+f\left(S_{u}+T_{z z} \cos (\lambda) / w\right) .
\end{aligned}
$$

The velocity-independent elements of the stiffness matrix are

$$
\begin{aligned}
& K_{0}(1,1)=g m_{t} z_{t}, \\
& K_{0}(1,2)=-g S_{u}, \\
& K_{0}(2,1)=K_{0}(1,2), \\
& K_{0}(2,2)=-g S_{u} \sin (\lambda),
\end{aligned}
$$

and the elements of the stiffness matrix to be multiplied by the square of the forward speed, $v^{2}$, are

$$
\begin{aligned}
& K_{2}(1,1)=0 \\
& K_{2}(1,2)=\left(S_{t}-m_{t} z_{t}\right) \cos (\lambda) / w \\
& K_{2}(2,1)=0 \\
& K_{2}(2,2)=\left(S_{u}+S_{f} \sin (\lambda)\right) \cos (\lambda) / w .
\end{aligned}
$$

\section{References}

1. Biral, F., Bortoluzzi, D., Cossalter, V., Da Lio, M.: Experimental study of motorcycle transfer functions for evaluating handling. Veh. Syst. Dyn. 39, 1-25 (2003)

2. Blue Team. http://www.roboticinfantry.com/

3. Cossalter, V., Lot, R., Maggio, F.: The modal analysis of a motorcycle in straight running and on a curve. Meccanica 39, 1-16 (2004)

4. Döhring, E.: Über die Stabilität und die Lenkkräfte von Einspurfahrzeugen. Ph.D. thesis, Technical University Braunschweig, Germany (1953)

5. Döhring, E.: Die Stabilität von Einspurfahrzeugen. Automob. Tech. Z. 56(3), 68-72 (1954)

6. Eaton, D.J.: Man-machine dynamics in the stabilization of single-track vehicles. Ph.D. thesis, University of Michigan (1973)

7. Jackson, A.W., Dragovan, M.: An experimental investigation of bicycle dynamics. Unpublished manuscript (1998)

8. Kooijman, J.D.G.: Experimental validation of a model for the motion of an uncontrolled bicycle. M.Sc. thesis, Delft University of Technology, April 2006. http://tam.cornell.edu/ als93/Koo06.pdf

9. Limebeer, D.J.N., Sharp, R.S.: Bicycles, motorcycles and models. IEEE Contr. Syst. Mag. 26(5), 34-61 (2006)

10. Miyagishi, S., Kageyama, I., Takama, K., Baba, M., Uchiyama, H.: Study on construction of a rider robot for two-wheeled vehicle. JSAE Rev. 24(3), 321-326 (2003)

11. Rice, R.S., Roland, R.D.: An evaluation of the performance and handling qualities of bicycles. Cornell Aero. Lab. Report No. VJ-2888-K (1970)

12. Rice, R.S.: Accident avoidance capabilities of motorcycles. In: Proceedings International Motorcycle Safety Conference, 16-17 December 1975, USDOT, pp. 121-134. National Highway Traffic Safety Administration, Washington (1975)

13. Roland, R.D.: Computer simulation of bicycle dynamics. In: Bleustein, J.L. (ed.) Mechanics and Sport. AMD, vol. 4, pp. 35-83. ASME, New York (1973) 
14. Roland, R.D., Lynch, J.P.: Bicycle dynamics, tire characteristics and rider modeling. Cornell Aero. Lab. Report No. YA-3063-K-2 (1972)

15. Roland, R.D., Massing, D.E.: A digital computer simulation of bicycle dynamics. Cornell Aero. Lab. Report No. YA-3063-K-1 (1971)

16. Ruijs, P.A.J., Pacejka, H.B.: Recent research in lateral dynamics of motorcycles. In: Proceedings of the 9th IAVSD Symposium held at Linköping University, Linköping, Sweden, 24-28 June 1985, pp. 467480 (1986)

17. Schwab, A.L., Meijaard, J.P., Papadopoulos, J.M.: Benchmark results on the linearized equations of motion of an uncontrolled bicycle. KSME Int. J. Mech. Sci. Technol. 19(1), 292-304 (2005)

18. Suhr, W.: Entwicklung und Erprobung eines nichtlinearen dynamischen Fahrradmodells, excerpts from a Ph.D. thesis, University of Oldenburg, Germany (1992)

19. Video recording of a test run. http://tam.cornell.edu/ als93/bicycledyn.htm\#May24

20. Wächter, M.: Eine experimentelle Überprüfung des dynamischen Fahrradmodells. M.Sc. thesis, University of Oldenburg, Germany (1991)

21. Weir, D.H., Zellner, J.W.: Lateral-directional motorcycle dynamics and rider control. Technical report No. 780304, Society of Automotive Engineers, Warrendale, PA (1978) 